\title{
TAFRO syndrome with a rapid fatal course despite corticosteroid and tocilizumab therapy
}

\author{
Hiroaki Tanaka $^{1}$ - Satoshi Bujo ${ }^{2}$ Ei Itobayashi ${ }^{3}$ - Akira Nakamura ${ }^{3}$ - Akihiro Ishii ${ }^{1}$. \\ Yoshio Suzuki ${ }^{4}$
}

Received: 27 December 2016 / Accepted: 24 January 2017 / Published online: 4 February 2017

(C) Springer-Verlag Berlin Heidelberg 2017

\begin{abstract}
TAFRO syndrome is a recently proposed subtype of multicentric Castleman's disease. Some patients have a refractory and aggressively fatal course. Here we report a case of TAFRO syndrome with a rapid fatal course despite corticosteroid and tocilizumab therapy. A 68-yearold Japanese male suffered from abdominal pain, watery diarrhea, fever, and general fatigue. On the 12th hospital day, severe thrombocytopenia, anasarca, and renal failure developed. The histopathological findings of lymph node biopsy were indicative of MCD. Although corticosteroid and tocilizumab were administered, the patient died of multiple organ failure on the 30th hospital day. Autopsy findings revealed that inflammation due to TAFRO syndrome was suppressed but multiple infarctions progressed and became the direct cause of death. Our case suggested that TAFRO syndrome should not be excluded even if clinical findings are insufficient for the diagnostic criteria at the onset and that in patients with TAFRO syndrome who are at a high risk of thrombosis, multiple infarctions may become a direct cause of death.
\end{abstract}

Hiroaki Tanaka

htanaka@hospital.asahi.chiba.jp

1 Department of Hematology, Asahi General Hospital, I-1326, Asahi, Chiba 289-2511, Japan

2 Department of Cardiovascular Medicine, Asahi General Hospital, Asahi, Chiba, Japan

3 Department of Gastroenterology, Asahi General Hospital, Asahi, Chiba, Japan

4 Department of Diagnostic Pathology, Asahi General Hospital, Asahi, Chiba, Japan
Keywords TAFRO syndrome $\cdot$ Castleman's disease · Tocilizumab $\cdot$ Multiple infarctions $\cdot$ Abdominal pain

\section{Introduction}

Castleman's disease (CD) is a rare form of lymphoproliferative disorder that is diagnosed by pathological findings. Histologically, the affected lymph tissues are classified as hyaline-vascular (HV) type, plasma-cell (PC) type, and a mixed-type variant of the two [1]. High interleukin (IL)-6 levels are closely related to this disease. In addition, CD is divided into two clinical subtypes: unicentric variant $\mathrm{CD}$ and systematic or multicentric variant CD (MCD) [2].

TAFRO syndrome is a recently proposed subtype of MCD [3-5], which is characterized by various symptoms, namely thrombocytopenia; anasarca, including pleural effusion and ascites; fever; reticulin fibrosis (myelofibrosis); renal insufficiency; and organomegaly, including hepatosplenomegaly and lymphadenopathy. Lymph node histology in most patients reveals mixed-type $\mathrm{CD}$, with few patients having HV-type MCD histology. However, compared with patients with MCD, patients with TAFRO syndrome present with atypical findings such as normal immunoglobulin levels, thrombocytopenia, relatively small lymphadenopathy, marked pleural effusion, ascites, and edema, in addition to an acute or subacute onset and clinical course [5]. These clinicopathologic discrepancies suggested that TAFRO syndrome is a distinct new entity rather than a subtype of MCD [6]. Similar to patients with MCD, some patients with TAFRO syndrome are sensitive to corticosteroids and anti- IL-6 receptor antibody (tocilizumab, TCZ) [6-8]; however, other patients are refractory to these treatments and exhibit an aggressively fatal course [9-13]. 
Here we report a case of a patient with TAFRO syndrome who died of multiple organ failure on the 30th hospital day despite prednisolone and TCZ treatment.

\section{Clinical history}

A 68-year-old Japanese male who was noted to have hypertension, diabetes mellitus, and dyslipidemia by periodic medical examinations visited the emergency department of our hospital for upper abdominal pain. Treatment for peptic ulcer did not resolve his symptoms. Upper gastrointestinal endoscopy and abdominal ultrasonography were performed; however, only mild splenomegaly was observed and there were no signs of dilatation or increased wall thickness in the intestinal tract as well as ascites. Whole-body enhanced computed tomography (CT) showed no findings regarding the cause of abdominal pain, except for mild cervical lymphadenopathy. Abdominal pain improved soon after the initiation of antibiotic therapy; however, watery diarrhea, fever, and general fatigue developed and were exacerbated. On January 2013, the patient was emergently hospitalized.

On admission, his height and weight were $167.6 \mathrm{~cm}$ and $81.7 \mathrm{~kg}$ (body mass index, 28.9), respectively. His vital signs were as follows: temperature, $37.5^{\circ} \mathrm{C}$; heart rate, $104 \mathrm{bpm}$; blood pressure, 194/112 $\mathrm{mmHg}$; and respiratory rate, 20 24 breaths $/ \mathrm{min}$. His oxygen saturation was $100 \%$ while breathing ambient air. Breathing rate and heart beats were normal. His abdomen was soft but distended due to obesity. Liver and spleen were not palpable. Bowel sounds were normal. His superficial lymph nodes were not palpable. Lower extremities were not edematous. There were no skin lesions. No particular abnormalities were detected by neurological examination.

The laboratory findings were showed in Table 1. Lower intestinal endoscopy did not reveal any finding suggestive of pseudomembranous enterocolitis. Despite broad spectrum antibiotic therapy, fever and elevated C-reactive protein levels did not improve. Exacerbation of abdominal distention, general edema, and oliguria were observed. Hypoalbuminemia and renal dysfunction gradually deteriorated. Positron emission tomography (PET)-CT revealed abnormal accumulation in the bilateral cervical and supraclavicular lymph nodes and left subclavian lymph nodes (Fig. 1). Whole-body enhanced CT performed on the 11th hospital day revealed massive pleural effusion and ascites. Considering the possibility of sarcoidosis, malignant lymphoma, or vasculitis syndrome, $1 \mathrm{mg} / \mathrm{kg}$ of prednisolone was initiated after cervical lymph node biopsy.

Lymph node biopsy showed blurred follicles with atrophic germinal centers. The peripheries of the follicles were surrounded by small lymphocytes that were tightly layered in a concentric manner, resulting in an onion-skin appearance (Fig. 2a, b). The inter-follicular area had increased plasma cell proliferation and vascularity, partially radiating from the germinal center (Fig. 2c, d). These histopathological findings were indicative of mixed-type CD. CD21 was positive for germinal center, but rarely stained inter-follicular area, excluding angioimmunoblastic T-cell lymphoma.

Because acute renal failure, pulmonary edema, and hyperkalemia were observed, emergency dialysis was started on the 15th hospital day. The laboratory data at that time were as follows: white blood cell count, $24.6 \times 10^{9} / \mathrm{L}$; hemoglobin, $10.1 \mathrm{~g} / \mathrm{dL}$; platelet count, $20.0 \times 10^{9} / \mathrm{L}$; total protein, $5.0 \mathrm{~g} / \mathrm{dL}$; albumin, $1.5 \mathrm{~g} / \mathrm{dL}$; and serum creatinine, $5.48 \mathrm{mg} / \mathrm{dL}$. Based on the pathological diagnosis of mixed-type $\mathrm{CD}, 8 \mathrm{mg} / \mathrm{kg}$ of TCZ was administered on the 22nd hospital day. However, the clinical status of the patient did not improve, and he died of heart and respiratory failure on the 30th hospital day.

\section{Materials and methods}

Autopsy was performed with the consent of his family. Hematoxylin and eosin staining sections and immunohistochemistry sections were prepared from formalin-fixed, paraffin-embedded tissue. Commercially available antibodies were used for immunohistochemistry.

\section{Results}

Anasarca was prominent with increased body weight (101.2 kg). Pleural effusion (right lung, $600 \mathrm{~mL}$; left lung, $1000 \mathrm{~mL})$, ascites $(3000 \mathrm{~mL})$, and pericardial effusion $(15 \mathrm{~mL})$ were found with mild fibrinous serositis. Multiple lymphadenopathies (diameter, $0.5-3.0 \mathrm{~cm}$ ) in cervical, mediastinal, para-aortic abdominal, and mesenteric lymph nodes were confirmed. Microscopic findings revealed the disappearance of lymphoid follicles and significant decrease in the number of plasma cells compared with the lymph node biopsy specimen (Fig. 3a-c). The bone marrow was hyperplastic with increased megakaryocytes and reticulin fibers (Fig. 3d, e). Infarctions of the kidney, liver, spleen, and digestive tract were found without finding suggesting vasculitis (Fig.3f-j). On autopsy, it was identified that renal and hepatic infarctions due to circulation failure were the direct causes of death.

\section{Discussion}

At the time of admission, our case did not meet the diagnostic criteria for TAFRO syndrome proposed by Masaki et al. in 2015 [5]. However, severe thrombocytopenia, anasarca, and renal failure developed on the 12th hospital day. The histopathological findings of lymph node biopsy were indicative of MCD. The autopsy finally revealed myelofibrosis. Our case suggested that TAFRO syndrome should not be excluded even if clinical findings are insufficient for the diagnostic criteria at 
Table 1 Laboratory data on admission

\begin{tabular}{|c|c|c|c|c|c|}
\hline \multicolumn{2}{|c|}{ Complete blood cell count } & \multicolumn{2}{|l|}{ Blood chemistry } & \multicolumn{2}{|l|}{ Serological test } \\
\hline White blood cell & $10.2 \times 10^{9} / \mathrm{L}$ & Total protein & $5.8 \mathrm{~g} / \mathrm{dl}$ & C-reactive protein & $24.92 \mathrm{mg} / \mathrm{dl}$ \\
\hline Neutrophil & $79.6 \%$ & Albumin & $2.6 \mathrm{~g} / \mathrm{dl}$ & $\operatorname{IgG}$ & $958 \mathrm{mg} / \mathrm{dl}$ \\
\hline Lymphocyte & $12.3 \%$ & GOT & $27 \mathrm{U} / 1$ & $\operatorname{IgA}$ & $207 \mathrm{mg} / \mathrm{dl}$ \\
\hline Monocyte & $7.2 \%$ & GPT & $20 \mathrm{U} / 1$ & $\operatorname{IgM}$ & $31 \mathrm{mg} / \mathrm{dl}$ \\
\hline Basophil & $0.1 \%$ & $\mathrm{LDH}$ & $157 \mathrm{U} / \mathrm{L}$ & IgG4 & $<3.0 \mathrm{mg} / \mathrm{dl}$ \\
\hline Eosinophil & $0.8 \%$ & ALP & $545 \mathrm{U} / \mathrm{L}$ & M-protein & $(-)$ \\
\hline Hemoglobin & $12.9 \mathrm{~g} / \mathrm{dl}$ & Total bilirubin & $0.9 \mathrm{mg} / \mathrm{dl}$ & $\mathrm{C} 3$ & $69 \mathrm{mg} / \mathrm{dl}$ \\
\hline Hematocrit & $40.1 \%$ & Blood urea nitrogen & $22 \mathrm{mg} / \mathrm{dl}$ & $\mathrm{C} 4$ & $16 \mathrm{mg} / \mathrm{dl}$ \\
\hline \multirow[t]{2}{*}{ Platelet count } & \multirow[t]{2}{*}{$186.0 \times 10^{9} / \mathrm{L}$} & Creatinine & $1.07 \mathrm{mg} / \mathrm{ml}$ & Antinuclear antibody & $(-)$ \\
\hline & & Ferritine & $346 \mathrm{ng} / \mathrm{ml}$ & Rheumatoid factor & $<5.0 \mathrm{IU} / \mathrm{ml}$ \\
\hline \multicolumn{2}{|l|}{ Coagulation test } & ACE & $5.1 \mathrm{U} / \mathrm{L}$ & PR3-ANCA & $<1.0 \mathrm{U} / \mathrm{ml}$ \\
\hline PT & $14.0 \mathrm{~s}$ & Lysozyme & $16.3 \mu \mathrm{g} / \mathrm{ml}$ & MPO-ANCA & $<1.0 \mathrm{U} / \mathrm{ml}$ \\
\hline PT-INR & 1.25 & IL-6 & $80.3 \mathrm{pg} / \mathrm{ml}$ & Soluble IL-2 receptor & $1020 \mathrm{U} / \mathrm{ml}$ \\
\hline APTT & $44.3 \mathrm{~s}$ & $\mathrm{HbA1c}$ & $6.8 \%$ & Hepatitis B surface antigen & $(-)$ \\
\hline Fibrinogen & $519 \mathrm{mg} / \mathrm{dl}$ & VEGF & $127 \mathrm{pg} / \mathrm{ml}$ & Hepatitis $\mathrm{C}$ virus antibody & $(-)$ \\
\hline D-dimer & $11.1 \mu \mathrm{g} / \mathrm{ml}$ & & & HIV antibody & $(-)$ \\
\hline \multicolumn{6}{|c|}{ Infection-associated test } \\
\hline \multicolumn{2}{|l|}{ Gaffky scale } & 0 & Aspergillus antigen & \multicolumn{2}{|l|}{$(-)$} \\
\hline \multicolumn{2}{|c|}{ TB-specific IFN- $\gamma$ release assay } & Negative & Candida antigen & \multicolumn{2}{|l|}{$(-)$} \\
\hline \multicolumn{2}{|c|}{ PCR assay of TB/MAC DNA } & Negative & $\begin{array}{l}\text { Cryptococcus neoformans } \\
\text { antigen }\end{array}$ & \multicolumn{2}{|l|}{$(-)$} \\
\hline \multicolumn{2}{|l|}{$\beta$-D-glucan } & $<4.0 \mathrm{pg} / \mathrm{ml}$ & Blood culture (2 sets) & \multicolumn{2}{|l|}{ Negative } \\
\hline
\end{tabular}

$P T-I N R$ intenational normalized ratio, APTT activated partial thromboplastin, ACE angiotensin-converting enzyme, VEGF vascular endothelial growth factor, $A N C A$ anti-neutrophil cytoplasmic antibody, $H I V$ human immunodeficiency virus, $T B$ mycobacterium tuberculosis, $I F N$ interferon, $P C R$ polymerase chain reaction analysis, MAC mycobacterium avium complex

the admission. Because myelofibrosis is a relatively specific finding of TAFRO syndrome, and bone marrow biopsy should always be performed.
A review of 12 patients with TAFRO syndrome treated with TCZ revealed that all patients, except one, were alive despite short observation periods [7]. Although our patient

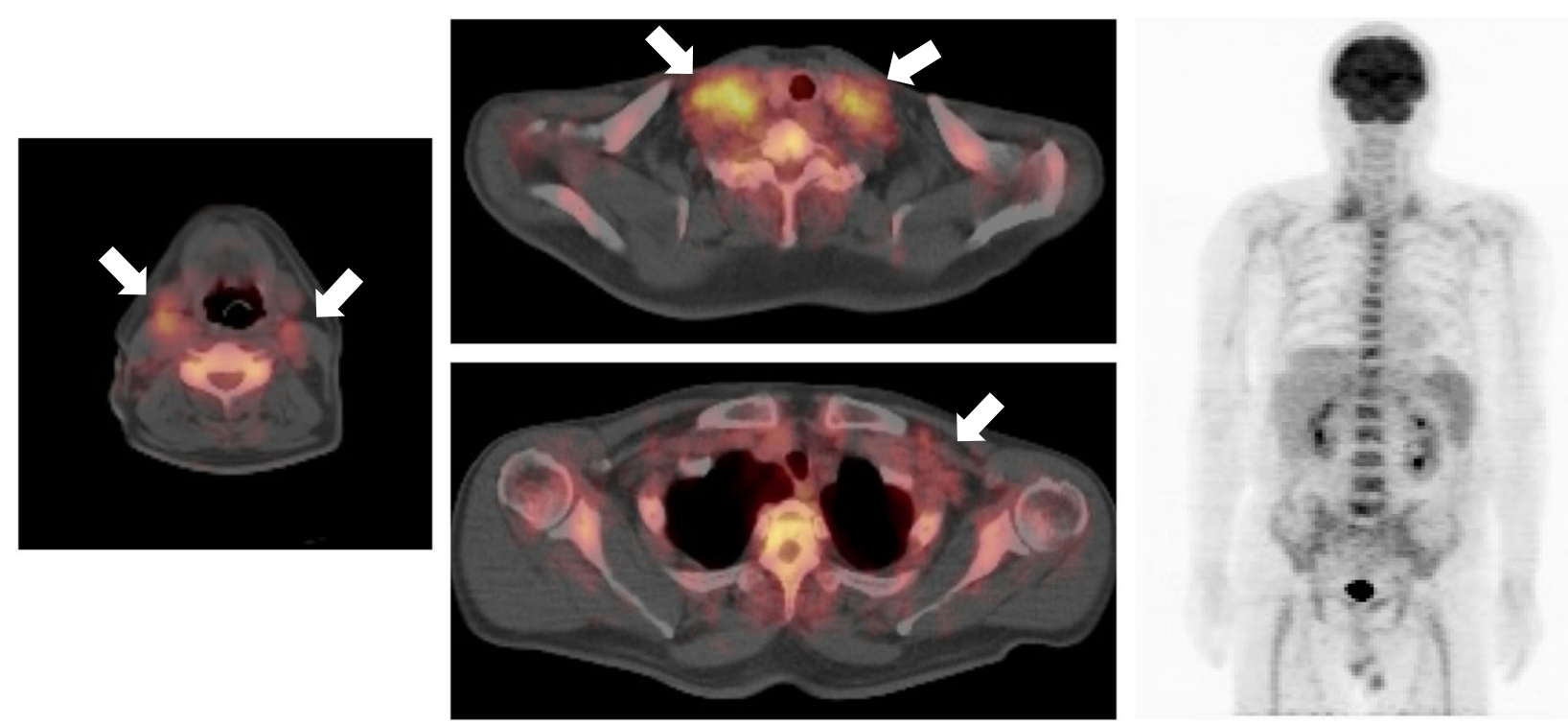

Fig. 1 Positron emission tomography-computed tomography showing abnormal accumulations in bilateral cervical and supraclavicular lymph nodes and left subclavian lymph nodes (arrows) 
Fig. 2 Pathological findings of the cervical lymph node biopsy. a Lymph node biopsy showing blurred follicles with atrophic germinal center (hematoxylin and eosin staining, $\times 100$ ). b

Peripheries of the follicles are surrounded by small lymphocytes that are tightly layered in a concentric manner, resulting in an onion-skin appearance $(\times 400)$. c, d Inter-follicular area with increased plasma cells and hyper-vascularity (c, $\times 400$; d, CD138 staining, $\times 400$ )
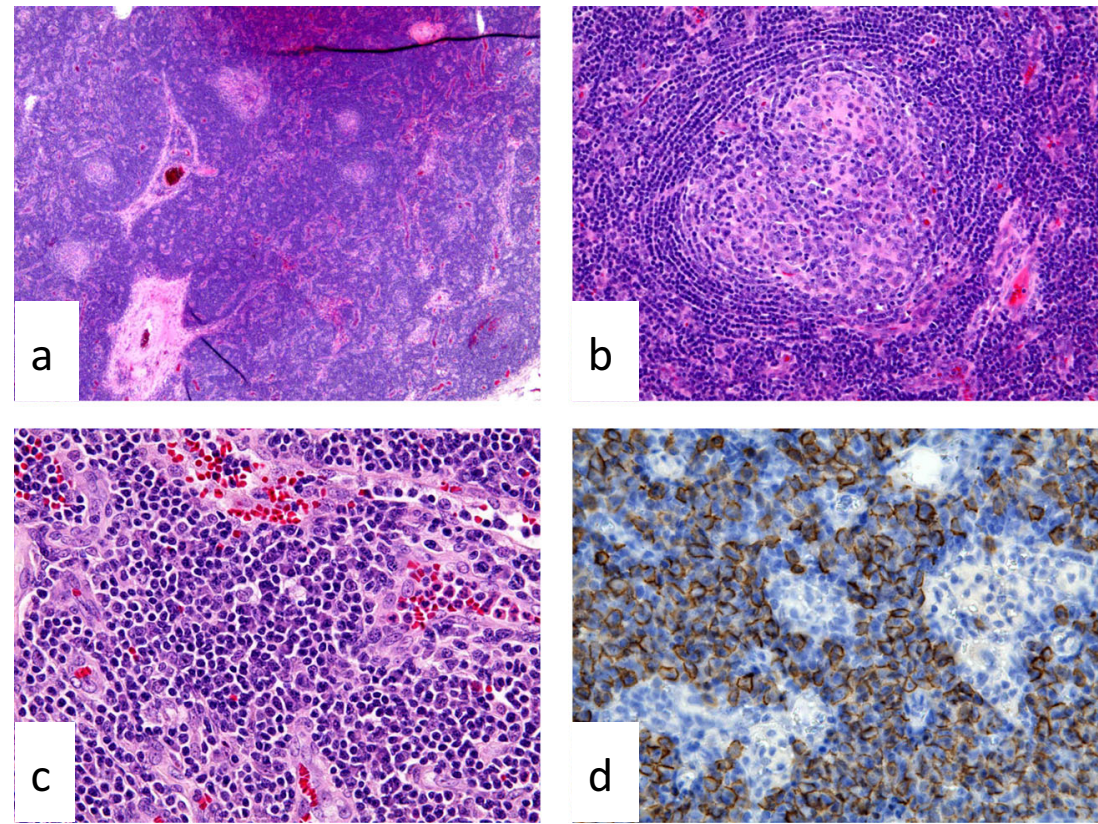

died of multiple organ failure despite TCZ treatment, autopsy findings suggested that inflammation due to TAFRO syndrome was suppressed by corticosteroid and TCZ treatment. The direct cause of his death was considered renal and hepatic infarction due to circulation failure. Tadokoro et al. reported a case of a patient with TAFRO syndrome who died of multiple organ failure 2 weeks after the initiation of TCZ therapy. Autopsy revealed infarction of the brain and ascending colon, suggesting that TAFRO caused excessive vascular permeability, leading to severe hypovolemia and subsequent organ failure and death [12]. This patient had a 4-year history of hypertension and dyslipidemia. Our patient also had risk factors of arteriosclerosis, such as hypertension, diabetes mellitus, dyslipidemia, and a long history of smoking. In patients with TAFRO syndrome who are at a high risk of thrombosis, multiple infarctions may become a direct cause of death. In a case report, Fujiwara et al. suggested that hypercytokinemia in TAFRO syndrome inhibits a disintegrin and a metalloproteinase with a thrombospondin type 1 motif, member 13 (ADAMTS13) activity, thereby inducing thrombotic microangiopathy [7]. In our case, because inflammation due to TAFRO syndrome had improved at the time of autopsy, it could not be known whether hypercytokinemia in TAFRO syndrome influenced his multiple infarctions.

Another notable finding in the present case is abdominal pain as the initial symptom. Extensive evaluation by enhanced CT, abdominal ultrasonography, and upper gastrointestinal endoscopy did not reveal any findings suggestive of gastrointestinal ulceration, ischemic bowel disease, or ascites. In a review of 25 patients with TAFRO syndrome, eight patients were reported to have abdominal pain [14]. Additionally, Hiramatsu et al. described several patients with TAFRO syndrome who had
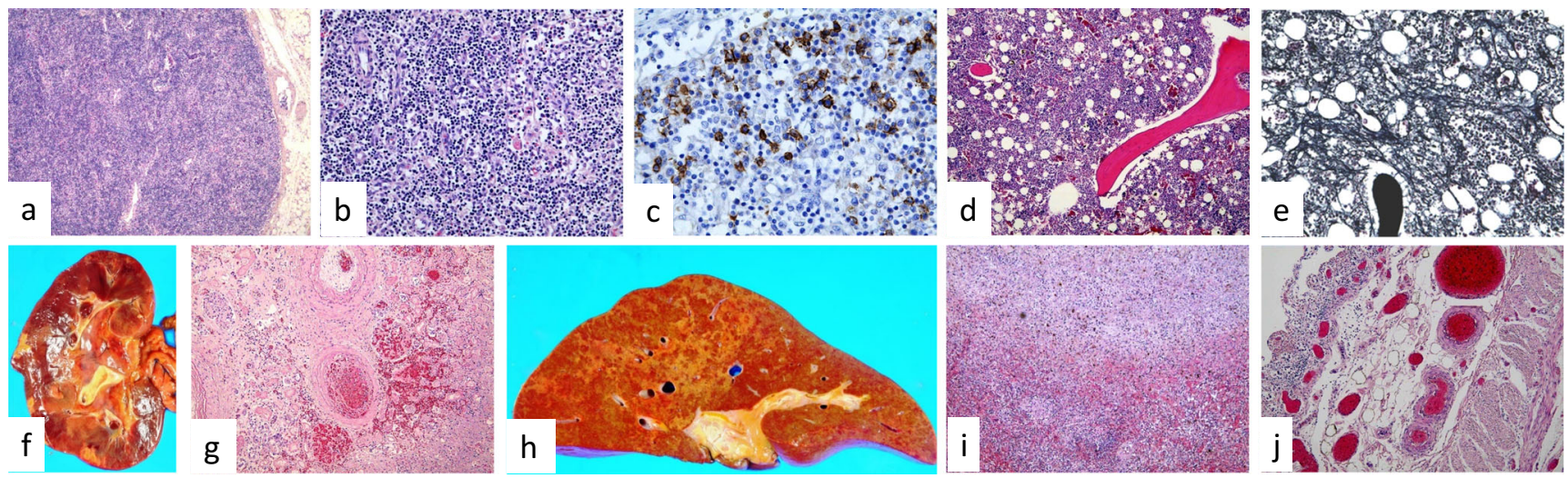

Fig. 3 a-c Pathological findings of autopsy. Disappearance of lymphoid follicle structures and significant decrease in the number of plasma cells compared with the lymph node biopsy specimen $(\mathbf{a}, \times 100 ; \mathbf{b}, \times 400$; , CD138 staining, $\times 400)$. d and e Bone marrow is hyperplastic with

increased megakaryocytes and reticulin fibers $(\mathbf{d}, \times 100$; e, silver impregnation staining). $\mathbf{f}-\mathbf{j}$. Infarctions of the kidney (f; $\mathbf{g}, \times 100)$, liver $(\mathbf{h})$, spleen $(\mathbf{i}, \times 100)$, and digestive tract $(\mathbf{j}, \times 100)$ were found 
abdominal pain as the initial symptom, with a few ascites [15]. Thus, abdominal pain may be a symptom to be noted as the initial symptom of TAFRO syndrome, even if there are few ascites.

In conclusion, we report a case of TAFRO syndrome with a rapid fatal course despite corticosteroid and TCZ treatment. Autopsy findings revealed that inflammation due to TAFRO syndrome was suppressed with these therapeutics; however, multiple infarctions due to circulation failure become the direct cause of death. Our case suggested that TAFRO syndrome should not be excluded even if clinical findings are insufficient for the diagnostic criteria at the onset and that in patients with TAFRO syndrome who are at a high risk of thrombosis, multiple infarctions may become a direct cause of death.

\section{Compliance with ethical standards}

Conflicts of interest The authors declare that they have no conflict of interest.

\section{References}

1. Frizzera G, Peterson BA, Bayrd ED, Goldman A (1985) A systemic lymphoproliferative disorder with morphologic features of Castleman's disease: clinical findings and clinicopathologic correlations in 15 patients. J Clin Oncol 3:1202-1216

2. El-Osta HE, Kurzrock R (2011) Castleman's disease: from basic mechanisms to molecular therapeutics. Oncologist 16:497-511

3. Takai K, Nikkuni K, Shibuya H, Hashidate H (2010) Thrombocytopenia with mild bone marrow fibrosis accompanied by fever, pleural effusion, ascites and hepatosplenomegaly. Rinsho Ketsueki 51:320-325

4. Kawabata H, Takai K, Kojima M et al (2013) Castleman-Kojima disease (TAFRO syndrome): a novel systemic inflammatory disease characterized by a constellation of symptoms, namely, thrombocytopenia, ascites (anasarca), microcytic anemia, myelofibrosis, renal dysfunction, and organomegaly: a status report and summary of Fukushima (6 June, 2012) and Nagoya meetings (22 September, 2012). J Clin Exp Hematop 53:57-61

5. Masaki Y, Kawabata H, Takai K et al (2016) Proposed diagnostic criteria, disease severity classification and treatment strategy for TAFRO syndrome, 2015 version. Int J Hematol 103:686-692

6. Iwaki N, Fajgenbaum DC, Nabel CS et al (2016) Clinicopathologic analysis of TAFRO syndrome demonstrates a distinct subtype of HHV-8-negative multicentric Castleman disease. Am J Hematol 91:220-226

7. Fujiwara $\mathrm{S}$, Mochinaga $\mathrm{H}$, Nakata $\mathrm{H}$ et al (2016) Successful treatment of TAFRO syndrome, a variant type of multicentric Castleman disease with thrombotic microangiopathy, with anti-IL6 receptor antibody and steroids. Int J Hematol 103:718-723

8. Sakai K, Maeda T, Kuriyama A, Shimada N, Notohara K, Ueda Y (2016) TAFRO syndrome successfully treated with tocilizumab: a case report and systematic review. Mod Rheumatol 17:1-6

9. Kawabata H, Kotani S, Matsumura Y et al (2013) Successful treatment of a patient with multicentric Castleman's disease who presented with thrombocytopenia, ascites, renal failure and myelofibrosis using tocilizumab, an anti-interleukin-6 receptor antibody. Intern Med 52:1503-1507

10. Fajgenbaum DC, van Rhee F, Nabel CS (2014) HHV-8-negative, idiopathic multicentric Castleman disease: novel insights into biology, pathogenesis, and therapy. Blood 123:2924-2933

11. Takai K, Nikkuni K, Momoi A, Nagai K, Igarashi N, Saeki T (2013) Thrombocytopenia with reticulin fibrosis accompanied by fever, anasarca and hepatosplenomegaly: a clinical report of five cases. J Clin Exp Hematop 53:63-68

12. Tadokoro A, Kanaji N, Hara T et al (2016) An uncharted constellation: TAFRO syndrome. Am J Med 129:938-941

13. Masaki Y, Nakajima A, Iwao H et al (2013) Japanese variant of multicentric castleman's disease associated with serositis and thrombocytopenia - a report of two cases: is TAFRO syndrome (Castleman-Kojima disease) a distinct clinicopathological entity? J Clin Exp Hematop 53:79-85

14. Yamaga Y, Tokuyama K, Kato T et al (2016) Successful treatment with cyclosporin A in tocilizumab-resistant TAFRO syndrome. Intern Med 55:185-190

15. Hiramatsu S, Ohmura K, Tsuji H et al (2016) Successful treatment by rituximab in a patient with TAFRO syndrome with cardiomyopathy. Nihon Rinsho Meneki Gakkai Kaishi 39:64-71 